\title{
Duftende Ananaslilien - Blütenduft als Schlüsselmerkmal für die Anlockung bestäubender Kleinsäuger
}

\author{
Petra Wester
}

\begin{abstract}
The flowers of the South African pineapple lily Eucomis regia (Asparagaceae) are pollinated by mice and elephant-shrews. All twelve representatives of the pineapple lilies (Eucomis) look similar regarding shape and colour of their inflorescences and flowers and in the presence of easily accessible nectar. Key attractant for pollinators is floral scent, differing heavily in carrion fly-pollinated and wasp-pollinated Eucomis species as well as in E. regia. The floral odour of E. regia and one of its main compounds, the sulphur compound methional with a boiled-potato-like smell, was highly attractive to mice and elephant-shrews.
\end{abstract}

\section{Zusammenfassung}

Die Blüten der südafrikanischen Ananaslilie Eucomis regia (Asparagaceae) werden von Mäusen und Rüsselspringern bestäubt. Alle zwölf Arten der Ananaslilien (Eucomis) gleichen sich in Bezug auf Form und Farbe ihrer Blütenstände und Blüten sowie im Vorhandensein von leicht zugänglichem Nektar. Schlüsselmerkmal zur Anlockung von Bestäubern ist der Blütenduft, der sich bei Aasfliegen-bestäubten und Wegwespen-bestäubten Eucomis-Arten sowie bei E. regia stark unterscheidet. Der Duft von E. regia und eine seiner Hauptkomponenten, das schwefelhaltige, nach gekochten Kartoffeln duftende Methional, wirkt anlockend auf Mäuse und Rüsselspringer.

\section{Kleinsäuger bestäuben Eucomis regia}

Bestäubung von Blüten durch Kleinsäuger ist in Südafrika zwar kein häufiges, aber auch kein ungewöhnliches Phänomen (Wester 2009, 2011). Ein Beispiel ist Eucomis regia, ein Vertreter der zwölf Arten umfassenden afrikanischen Ananaslilien, die zu den Spargelgewächsen (Asparagaceae) gehört. Eucomis regia ist die einzige Art, die im Winterregengebiet, im Westen Südafrikas, vorkommt. Eucomis regia-Pflanzen wurden an verschiedenen Orten in Südafrika tagsüber und nachts direkt oder mit Hilfe von Videokameras beobachtet. Namaqua-Felsenmäuse (Micaelamys namaquensis) konnten nachts direkt beim Blütenbesuch beobachtet und fotografiert werden. Es war deutlich zu erkennen, dass die Mäuse gezielt zu den blühenden Pflanzen liefen, ihre Schnauze zwischen die Staubblätter der Blüten schoben und den Nektar mit ihrer Zunge aufleckten. Dabei berührten die Tiere die Pollensäcke und die Narbe der Blüten und wurden mit Pollen an ihrer Schnauze eingestäubt. Während des Blütenbesuchs beschädigten die Tiere die Blüten nicht. Auch auf den Videoaufnahmen waren Blütenbesuche durch NamaquaFelsenmäuse zu beobachten, aber ebenfalls durch Kap-Rüsselspringer (Elephantulus edwardii), die die Blüten auf die gleiche Art besuchten. Rüsselspringer sind nicht mit Mäusen (Ordnung: Na- getiere, Rodentia) verwandt, sondern gehören als Ordnung der Macroscelidea zu einer eigenen Gruppe, den Afrotheria, zu denen man auch Ele-

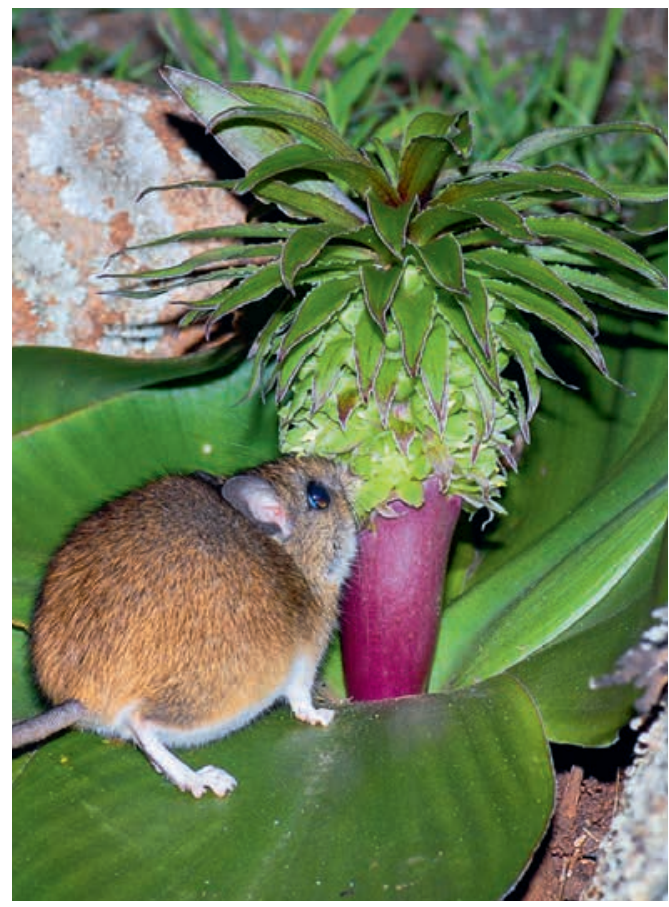

Abb. 1: Namaqua-Felsenmaus (Micaelamys namaquensis) trinkt Nektar aus Blüten der Ananaslilie Eucomis regia. (Foto: P. Wester) 


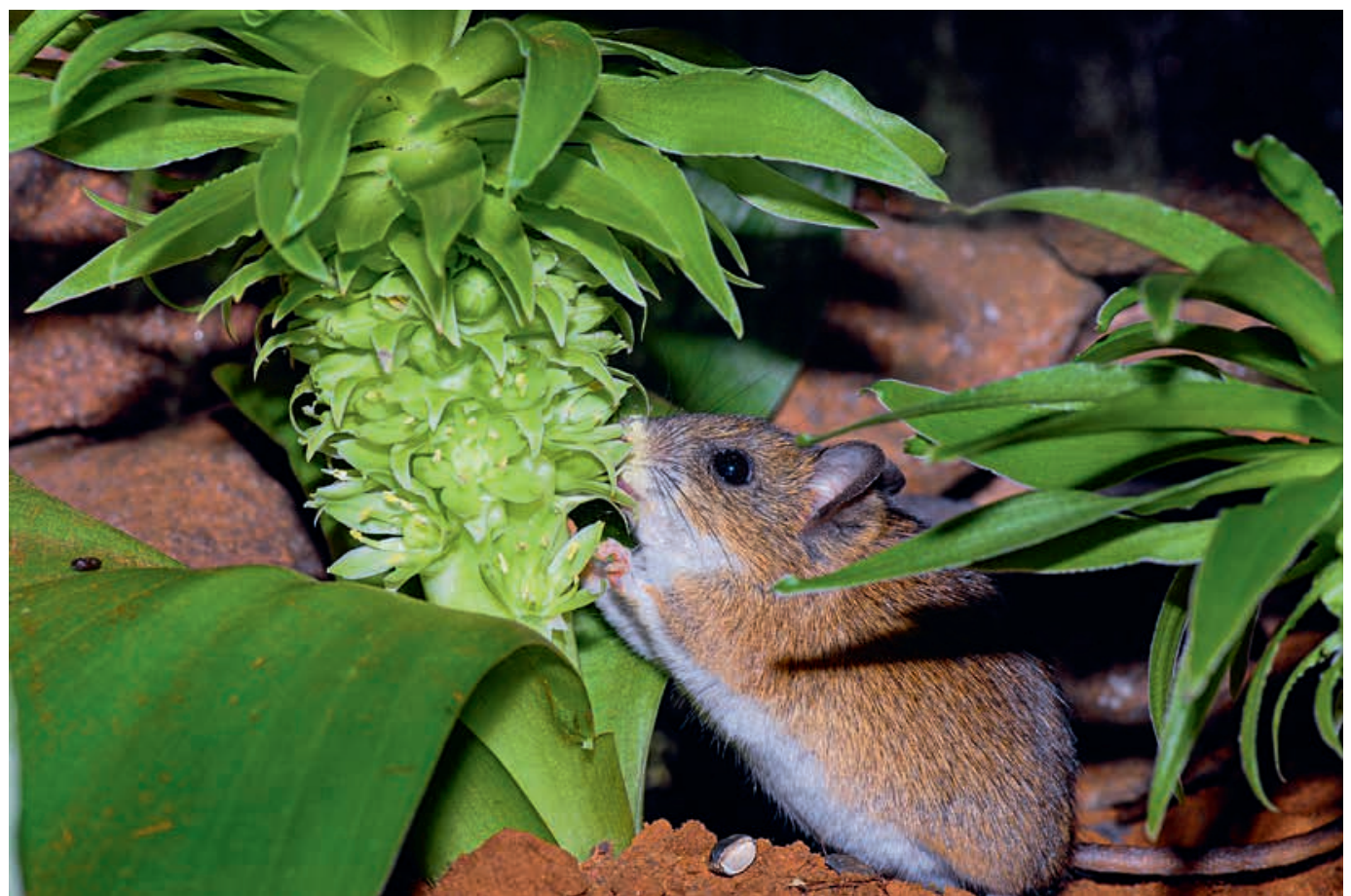

Abb. 2: Eine Namaqua-Felsenmaus leckt Nektar mit ihrer Zunge von E. regia-Blüten und wird dabei mit Pollen rund um ihre Schnauze eingestäubt. (Foto: P. WeSter)

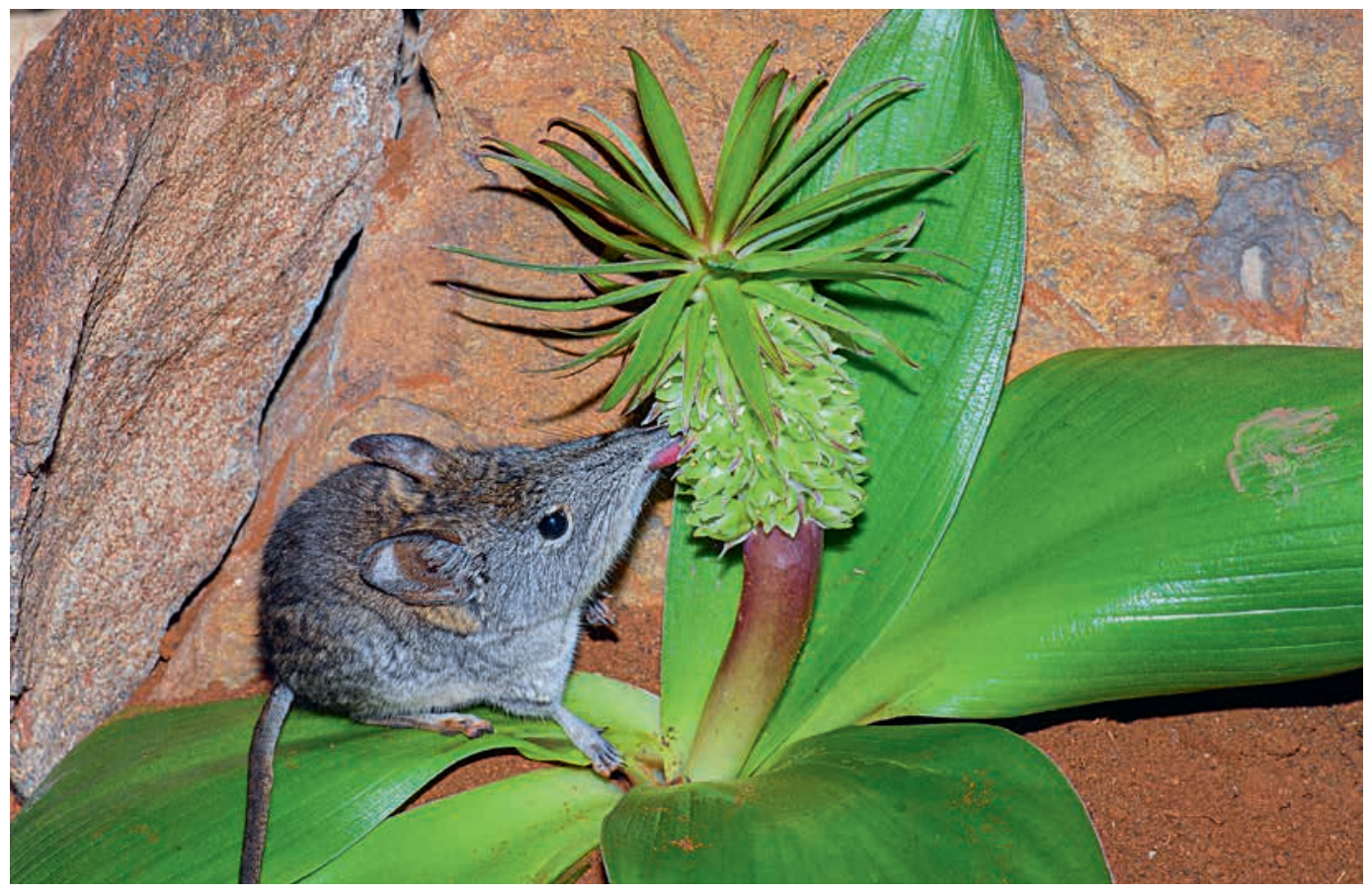

Abb. 3: Ein Kap-Rüsselspringer (Elephantulus edwardii) leckt Nektar mit seiner langen Zunge, dabei wird Pollen von E. regia-Blüten auf seiner Nase deponiert. (Foto: P. WeSTER) 
fanten, Seekühe, Erdferkel und andere Tiere zählt. Rüsselspringer wurden erst vor kurzem als Nektartrinker identifiziert (Wester 2010). Auch die Rüsselspringer berührten die Pollensäcke und die Narbe der E. regia-Blüten und wurden mit Pollen an ihrer langen, beweglichen Nase eingestäubt.

Im näheren Umkreis der blühenden Pflanzen wurden Lebendfallen aufgestellt, um Tiere auf Pollen an der Schnauze und im Kot zu testen, was Rückschlüsse auf Blütenbesuche zulässt. Dabei wurden neben den oben genannten Arten auch Vierstreifen-Grasmäuse (Rhabdomys pumilio) und Afrikanische Zwergmäuse (Mus minutoides) gefangen. Die Schnauzen-Abstriche und der Kot aller Tiere enthielten E. regia-Pollen in großer Menge. Vierstreifen-Grasmäusen, Zwergmäusen und den anderen Kleinsäugerarten wurden in Terrarien blühende E. regia-Pflanzen präsentiert. Die Tiere liefen zielgerichtet zu den Pflanzen, um Nektar zu trinken; sie zeigten das gleiche Verhalten wie im Gelände bzw. wie in den Videos zu sehen war.

Zusätzlich wurde bei Namaqua-Felsenmäusen, Vierstreifen-Grasmäusen und Rüsselspringern experimentell untersucht, ob sie Pollen zwischen verschiedenen Pflanzen transportieren können. Dies wurde simuliert, indem Pollensäcke einiger E. regia-Pflanzen mit Farbpuder eingestäubt und nach Blütenbesuchen die Narben anderer Pflanzen auf Pollenübertragung überprüft wurden. Es zeigte sich, dass alle Tiere den Farbpuder auf die Narben anderer E. regia-Pflanzen übertrugen, ein weiterer Hinweis, dass die Tiere Bestäuber von E. regia sind.

Außerdem wurde durch Abdecken der Pflanzen mit Drahtkäfigen (die selektiv Kleinsäuger ausschließen, aber potenziell Insekten passieren lassen) und anschließender Kontrolle des Samenansatzes im Vergleich zu nicht und komplett abgedeckten Pflanzen gezeigt, dass Kleinsäuger zur Samenproduktion von E. regia beitragen.

Die verschiedenen Untersuchungen zeigten, dass Kleinsäuger E. regia bestäuben, aber es stellt sich die Frage, wodurch die Tiere zum Blütenbesuch bei $E$. regia angelockt werden.

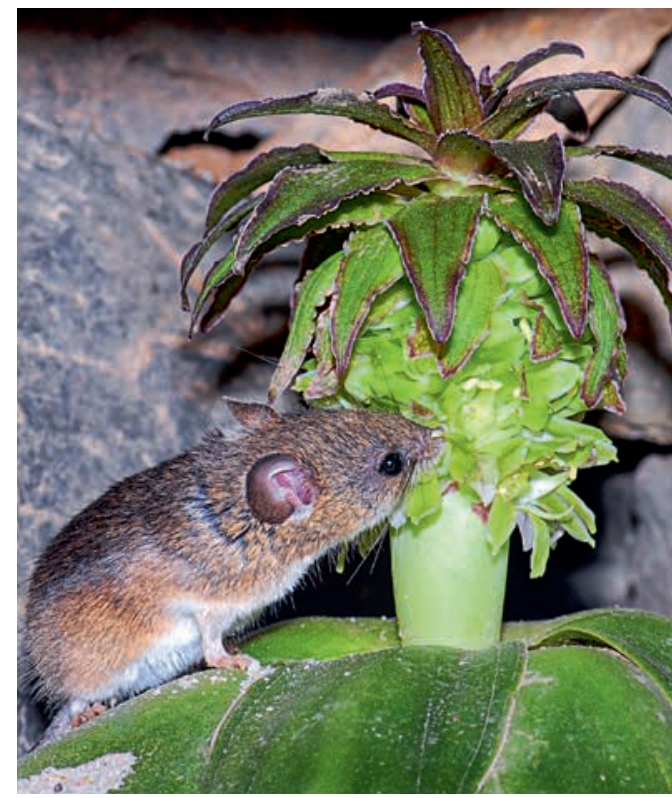

Abb. 4: Eine Zwergmaus (Mus minutoides) besucht eine Blüte von E. regia. Man sieht einen Pollensack auf der Schnauze der Maus. (Foto: P. Wester)

\section{Ananaslilien - scheinbar unattraktive Vertreter der Spargelgewächse}

Neben E. regia, die offensichtlich von Kleinsäugern bestäubt wird, gibt es Eucomis-Arten, die von Aasfliegen bestäubt werden (E. bicolor, E. humilis) und solche, die auf Wegwespen als Bestäuber spezialisiert sind (E. autumnalis, E. comosa) (Shuttleworth \& Johnson 2009, 2010). Morphologische Messungen und Farbmessungen ergaben, dass alle Eucomis-Arten sich in Form und Farbe ihres Blütenstandes und ihrer Blüten gleichen. Die grünlichen, unauffälligen Blüten aller Arten produzieren leicht zugänglichen Nektar.

\section{Blütenduft als Schlüsselattraktans für} Aasfliegen, Wegwespen und Kleinsäuger

Der Blütenduft unterscheidet sich stark bei den Aasfliegen-bestäubten und Wegwespen-bestäubten Eucomis-Arten sowie bei E. regia. Während der Duft der Wegwespen-bestäubten Arten als würzig beschrieben wird und der der Aasfliegen-bestäubten Eucomis-Arten als unangenehm stinkend (SнutTLEWORTH \& JoHNSON 2009, 2010), duftet E. regia nach gekochten Kartoffeln (Wester et al. 2019). Gesammelter Duft wurde mit Gaschromatografie- 


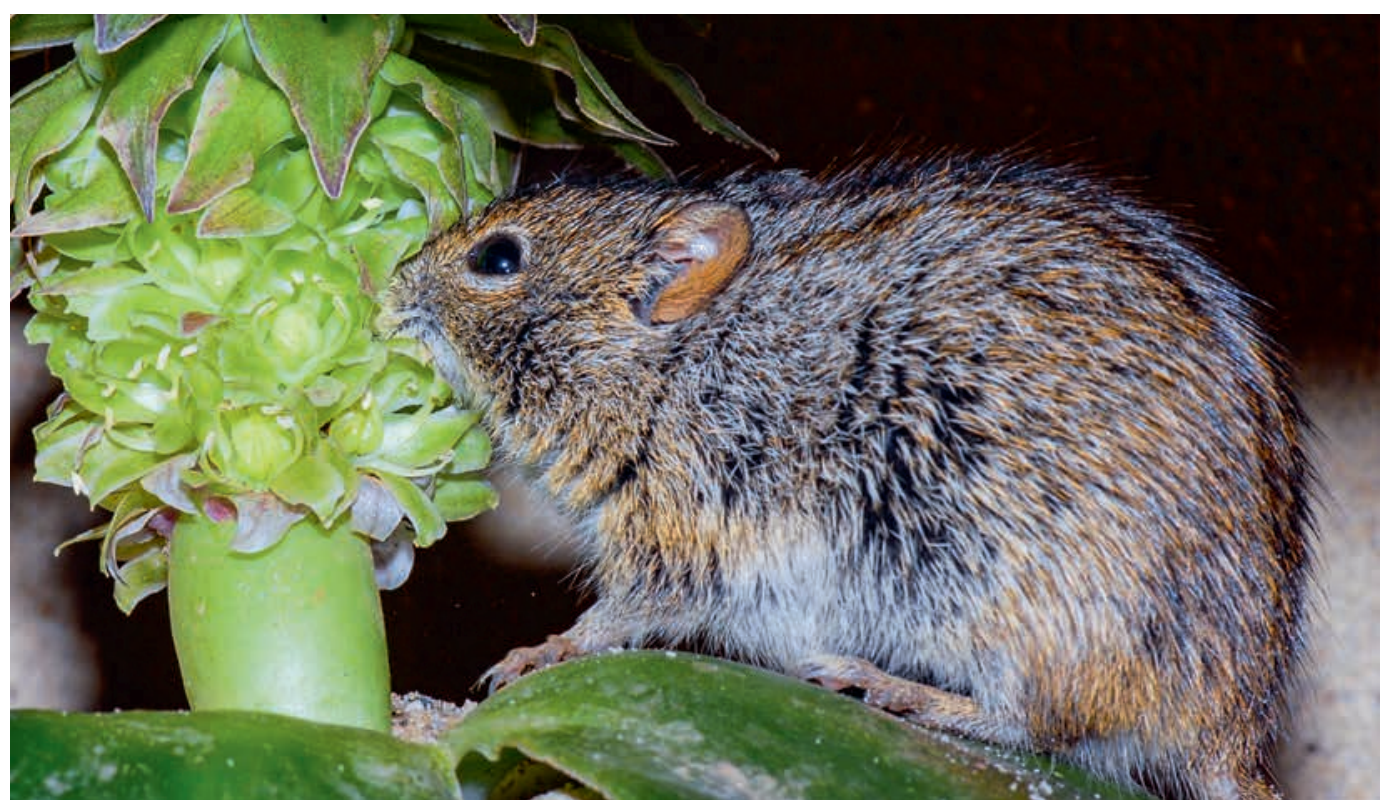

Abb. 5: Eine Vierstreifen-Grasmaus (Rhabdomys pumilio) presst ihre Schnauze in eine E. regia-Blüte. Man sieht einen Pollensack und Pollen auf der Schnauze. (Foto: P. Wester)

Massenspektrometrie untersucht. Die Ergebnisse bestätigten die Duftbeschreibungen; die Pflanzen unterscheiden sich in der Zusammensetzung ihrer Duftkomponenten. Hauptkomponente des Duftes von E. regia ist das schwefelhaltige Methional, welches für den Kartoffelduft verantwortlich ist. Methional entsteht auch beim Kochen von Kartoffeln (LANDAUd et al. 2009).

Auswahlversuche mit Mäusen und Rüsselspringern haben gezeigt, dass E. regia-Blütenduft und Methional auf die Tiere anlockend wirkt (Wester et al. 2019, Wester, unveröffentlichte Daten), nicht jedoch die Hauptkomponenten des Duftes der Aasfliegen-bestäubten und Wegwespenbestäubten Eucomis-Arten. Dies unterstützt die Annahme, dass nicht die visuellen Blütenmerkmale, sondern der Blütenduft von Eucomis, ein Schlüsselmerkmal zur Anlockung von Bestäubern ist.

\section{Literatur}

Landaud, S., Helinck, S. \& Bonnarme, P. 2008. Formation of volatile sulfur compounds and metabolism of methionine and other sulfur compounds in fermented food. Appl. Microbiol. Biotechnol. 77: 1191-1205.
Shuttleworth, A. \& Johnson, S. D. 2009. A key role for floral scent in a wasp-pollination system in Eucomis (Hyacinthaceae). - Ann. Bot. 103: 715-725.

Shuttleworth, A. \& Johnson, S. D. 2010. The missing stink: sulphur compounds can mediate a shift between fly and wasp pollination systems. - Proc. Royal Soc. B 277: 28112819.

Wester, P. 2009: Von Mäusen und Pavianschuhen - Erste Geländebeobachtungen von Nager-Bestäubung in Südafrika. Palmengarten 73: 120-125. DOI:https://doi.org/10.21248/ palmengarten.91

Wester, P. 2010: Sticky snack for Sengis: The Cape rock elephant-shrew, Elephantulus edwardii (Macroscelidea), as a pollinator of the Pagoda lily, Whiteheadia bifolia (Hyacinthaceae). Naturwissenschaften 97: 1107-1112.

Wester, P. 2011: Klebrige Nascherei für Rüsselspringer: Elephantulus edwardii als Bestäuber von Whiteheadia bifolia. Palmengarten 75: 122-125.

Wester, P., Johnson, S. D. \& Pauw, A. 2019: Scent chemistry is key in the evolutionary transition between insect and mammal pollination in African pineapple lilies. New Phytol. 222: 1624-1637.

\section{Anschrift der Autorin}

Dr. Petra Wester, School of Life Sciences, University of KwaZulu-Natal, P.B. X01, Pietermaritzburg 3209, Südafrika, E-Mail: westerpetra3@gmail.com https://orchid.org/0000-0002-6514-8159 